\title{
Blockchain Technology and Decentralized Governance: The Pitfalls of a Trustless Dream
}

\author{
Primavera De Filippi \\ CERSA/CNRS/Université Paris II \\ Berkman-Klein Center for Internet \& Society at Harvard
}

\section{Introduction}

Blockchain technology is considered by many as a highly disruptive technology that could trigger a new digital revolution (Tapscott \& Tapscott 2015). Indeed, by eliminating the need for a middleman, the technology has the potential to disrupt many sectors of activities that currently rely on a trusted authority or intermediary operator (Swan 2015).

Blockchain technology was first elaborated as a part of the Bitcoin network, a decentralized payment system and virtual currency that can be traded on a peer-to-peer network, without relying on banks or other financial institutions (Nakamoto, 2008). Deployed in 2009 by a pseudonymous entity called Satoshi Nakamoto, the idea underlying Bitcoin was that it is possible to delegate to a technological artefact the trust that we have thus far granted to existing social or political institutions. As such, the technology has been often referred to as "trustless technology" (Scott, 2015) or as a "trust machine" (The Economist, 2015)-because it eliminates the need to rely on trusted intermediaries, as long as one can trust the underlying technology (Brite \& Castillo, 2013).

When it comes to governance, the most significant innovation of blockchain technology is that it provides us with the ability to experiment with new organizational structures and distributed governance models, which are more transparent and less hierarchical than those we have become accustomed to. Indeed, the technology potentially allows for the creation of so-called decentralized blockchain-based organizations, enabling people to coordinate themselves in a peer-to-peer manner, according to a set of protocols and rules incorporated into self-executing smart contract code (Wright \& De Filippi, 2015).

Because it enables people to coordinate themselves in a peer-to-peer manner, early blockchain advocates claimed that blockchain technology would ultimately promote individual freedom and autonomy (Tapscott \& Tapscott 2015, Swan 2015, Atzori 2015). Yet, in practice, there are many limitations to that dream. As the Internet has shown, a decentralized infrastructure does not necessarily lead to a decentralization of powers within that infrastructure. To the contrary, over the years, the Internet network has grown more and more centralized, with a concentration of power in the hands of a few large online operators. Despite its promises of decentralization, the blockchain space is no exception to that. Over the years, the governance of the most popular blockchain networks has become highly centralized, and only a few large corporations (such as the main blockchain exchanges and wallet providers) are responsible for making blockchain technology accessible to the wider public. If we can learn anything from the history of Internet governance, it is crucial that we start thinking today about how we can shape the governance of blockchain-based networks in ways that ultimately preserve the distribution of powers of these infrastructures.

\section{A. The promises of decentralized collaboration}


Over the past two decades, the Internet has shown us that open and decentralized collaboration makes it possible to achieve things that would be really difficult to achieve internally, within a single company or organization (Raymond, 2001). Think of Wikipedia, for instance, which managed to compete with a large corporation like Microsoft, putting Encarta out of business in the matter of a few years and without any financial investment (Weber, 2004).

The success of this model has inspired a few economic players to experiment with new business models that rely on the power of the crowds in order to produce value (Brabham, 2013). This is the model adopted by most Internet giants, such as Facebook, Google or Twitter, as well as by new crowdsourcing platforms, such as Uber or AirBnb. If we observe the evolution of organizational structures over the past twenty years, we can observe a general trend, from centralized operators (e.g. newspapers, taxis or hotel operators) providing their services to a passive set of consumers, to centralized intermediaries (e.g. Facebook, Uber, or Airbnb) aggregating the contribution of a large number of people in order to provide a service to a much more active set of consumers.

Yet, the problem with the traditional crowdsourcing model is that, in most cases, the value produced by the crowd is not shared equally between the platform and the contributors (Kazeroun, 2014). It is instead concentrated into the hands of few large intermediaries, which ultimately dictate the rules of the platform (Brabham, 2012).

By eliminating the figure of the middleman, blockchain technology makes it possible to replace the model of top-down hierarchical organizations with a system of distributed and bottom-up cooperation. Instead of relying on a centralized operator or middleman, blockchain-based networks are designed to operate in a fully distributed manner, relying on a decentralized infrastructure to coordinate the interaction of all people contributing to these networks. Thanks to these new technology, we can thus witness the emergence of new decentralized crowd-sourcing organizations, which also aggregate the contribution of multiple people, but that do not require any kind of intermediary figure in order to manage the flow of contributions.

Bitcoin is perhaps the most representative example of a decentralized blockchain-based organization. Instead of relying on a centralized and hierarchical structure to operate the network, Bitcoin adopted a much more decentralized approach, inspired from the open source model. Anyone is free to contribute resources to the network, which operate thanks to the contributions of hundreds and thousands of people, collectively in charge of maintaining the network.

Over time, the model of decentralized collaboration adopted by Bitcoin has been applied to many other fields of activity, from cryptocurrencies and decentralized payment systems (e.g., Monero and Zerocash), to decentralized marketplaces (e.g. Open Bazaar and district0x), or even decentralized social networks (e.g. Steem.it and Akasha). These decentralized applications operate as decentralized blockchain-based organizations administrede by the community and for the community. Because there is no intermediary operator, every community member is simultaneously a contributor and an actual shareholder in the organisation. As a result, the value produced within these networks can-at least theoretically-be redistributed in a much more egalitarian way between all those who effectively contributed to the production of value.

\section{B. The limits of decentralized governance}


Despite their decentralized infrastructure, the governance of most blockchain-based networks or applications is highly centralized. Because of their pseudonymous nature, it is difficult to discern the actual degree of decentralization that characterizes these networks by simply looking at their protocol and operations. However, a more thorough investigation might reveal that the governance of these networks is oftentimes more centralized than what might seem at first glance.

The case of Bitcoin is an illustrative example of an underlying tendency towards the progressive concentration of powers within decentralized infrastructures (a tendency that can be observed in a large majority of blockchain-based applications). Designed as a perfectly decentralized system, the Bitcoin network operates in an autonomous and distributed manner, independently of any trusted authority or centralized operator. Yet, when we look more closely at the way Bitcoin operates, it does not take long to realize that its governance is actually quite centralized. On the one hand, there are only a few developers who have the necessary knowledge, expertise and power to decide on the evolution of the Bitcoin protocol (De Filippi \& Loveluck, 2016). On the other hand, although theoretically decentralized, the protocol for validating transactions (the so-called mining process) has today become highly concentrated into a few large mining pools, which currently control a large majority of the network. ${ }^{1}$

One of the main reason for this trend towards centralization is that is the mining process relies on a particular payoff structure governed by economic incentives (Proof of Work). While the system started as a fully decentralized network, after many years of operations, market dynamics (and economies of scale) eventually turned the network into a highly concentrated market, controlled by a few oligopolistic players-the so-called mining pools (Gervais \& al., 2014; Drescher, 2017).

Another interesting example of failure to achieve distributed governance is TheDAO, a decentralized blockchain-based investment fund deployed on the Ethereum blockchain, which was collective administered by a distributed network of peers. Launched in April 2016, TheDAO raised over \$160M worth of Ether in less than one month of existence. The governance mechanism was relatively simple. People who sent a particular amount of digital currency to the decentralized investment fund received a particular amount of tokens in exchange. These tokens enabled them to participate into the decision-making process concerning the fund's investments. While anyone could submit a proposal as to how they envision these funds to be spent, only the token holders could express their voice as to whether or not the proposal should be funded.

As a decentralized organization, TheDAO did not have any CEO or other leadership figure. Its governance structure was defined, only and exclusively, by the code deployed on the Ethereum blockchain, which precisely stipulated the procedures and rules that all members had to abide by. Yet, in spite of the decentralized nature of the fund, its governance structure was actually quite traditional. Indeed, as a decentralized blockchain-based organization, TheDAO was fundamentally governed as a plutocracy: the more money people had invested into fund, the greater their influence would be within the decision-making process.

Both of these examples illustrate one of key missing piece in the realm of blockchain governance. While we now have the opportunity to experiment with new decentralized infrastructures, we still have to conceive, design and implement a proper distributed governance system that could be

\footnotetext{
${ }^{1}$ As of June 2017, the four largest mining pools control almost $50 \%$ of the Bitcoin network, and more than $75 \%$ of the overall hashing power is controlled of only eight mining pools. For more information on Bitcoin's hashrate distribution, see https://blockchain.info/pools
} 
deployed top of this decentralized infrastructure. Of course, it is always possible to deploy a centralized governance system on top of a decentralized infrastructure, e.g. by assigning specific powers or privileges to a few identified individuals. Yet, if we want to fully enjoy the benefits of decentralization, it is necessary to come up with a decentralized model of governance.

Probably the closest thing to a decentralized governance system that we know of today is the free market-a system that relies on the emergent behavior of a distributed network of private players, following the laws of supply and demand in order to reach consensus as to what is the price of a particular good or service (Gale, 1955). Because of these properties, it could be argued that market dynamics operate according to a model of "distributed consensus"-similar to that of blockchains (Mattila, 2016).

The free market has, however, many limitations. First and foremost is the issue of transactionality. If the governance structure for decentralized blockchain-based networks or applications were based, only and exclusively, on market dynamics, many of the social interactions and noncommercial activities performed on top of these networks would ultimately turn into marketdriven transactions. Whether a blockchain is used for the purpose of trading or contracting, or whether it is used to facilitate coordination among a decentralized network of peers, the transactionality inherent in market-based governance systems might ultimately influence the underlying motivations of people interacting through the platforms-replacing ideological principles and social or relational motivations with economic rationality and instrumental logic.

Such a scenario had already been envisioned, over fifteen years ago, by French philosopher and media theorist Pierre Lévy, who saw the Internet as a tool that-if improperly used-could lead to a whole new market-oriented society. Lévy described the Internet as a new space that could become "both the ultimate place for the competition of ideas and a market finally freed from all constraints. There will be no more difference between thought and business. Money will reward the ideas most likely to bring about the best possible future-the future we will choose to buy." (Levy, 2000; p. 100)

To many of the self-declared crypto-libertarians that currently populate the blockchain space, this is not necessarily a bad thing. Indeed, according to some of the early blockchain advocates (Atzori, 2015), as the ideals of decentralization and free market blur into each other, blockchain-based applications and market-based governance systems could provide a more efficient way to govern society than reliance on the coercive force of government authorities.

Yet, as it has been shown in the past, without any governmental intervention, markets can be easily be manipulated by powerful actors (Posner, 1974)-and the law of supply and demand might not always lead to the most effective outcome for a democratic society (Stigler, 1971). Indeed, if and when blockchain technology will manage to impregnate itself into the very fabric of society, some of today's legal, social and political institutions might need to accommodate new technological constructs operated by market forces and code-based rules. While these systems might bring new promises of increased transparency and accountability, if improperly governed, we might incur the risk of losing some of the basic tenets of a democratic society.

\section{c. The end of a trustless dream}

Given these limitations to market-based governance, we might wonder whether blockchain technology can actually provide the necessary infrastructure for a more decentralized society. To 
be sure, by eliminating the figure of the middleman, blockchain technology allows for people to cooperate in a more decentralized manner. Yet, if we look at the real-world applications of the technology, we can see that they actually promote a very individualist approach to collaboration.

Often praised for their potential to support or facilitate a more collaborative economy, the current implementation of existing blockchain-based networks is often inconsistent with the original conception of the collaborative economy-defined as an economy based on "horizontal networks and democratic participation" (Botsman \& Rogers, 2010). In its original conception, the collaborative economy was not meant to rely on a centralized (trusted) authority responsible for mediating interactions between a distributed network of (untrusted) actors. Rather, players in the collaborative economy had to contribute towards building and validating trusted relationships between members of their communities (Hawlitschek \& al., 2016). Conversely, with a blockchain, trust is perceived as something that should be reduced to the maximum. Economic transactions and social interactions are mediated by a so-called "trustless system", where trust in people is replaced by trust in the underlying technological framework.

Accordingly, when it comes to decentralized cooperation and collaboration, one of the greatest feature of a blockchain-its trustless infrastructure-is also one of its greatest drawbacks. Specifically, the trustlessness of these systems actually lessen the opportunities for people to create strong social connections and community ties. Because people do not need to trust one another, they also do not need to create bonds between each other.

Most critically, because of the specific payoff structures embedded into a majority of these blockchain-based systems-which assume that everyone is a defector-these systems are generally designed to work in highly antagonistic environments, rather than collaborative ones (Kroll \& al., 2013). Hence, while the robustness of these systems is usually high, their efficiency is, however, significantly inferior to what it could have been, had they been designed to account for the fact that most people are more likely to cooperate than to defect.

For instance, although Bitcoin requires people to collaboratively participate to maintaining the network by contributing computing resources to the underlying blockchain network, it does not necessarily promote a collaborative environment. Quite to the contrary, the introduction of market-based incentives into the system is such as to distort the underlying motivations of people, who are ultimately brought to compete with each other (Sapirshtein \& al., 2015) in order to be the first to find the solution to the mathematical puzzle associated with each block and acquire the corresponding reward (Lewenberg \& al., 2015).

Any blockchain-based organization whose governance system relies-mainly or exclusively-on market dynamics is, therefore, ultimately bound to fail. On the one hand, the adoption of a marketdriven governance system is likely to usher in competitive dynamics that may significantly reduce the opportunities for people to cooperate around a common goal, ultimately harming the interests of the organization to which they contribute. On the other hand, without an institution protecting them, markets-albeit competitive at first-have a tendency to concentrate and eventually turn into oligopolistic market structures, ruled by a few dominant players.

Accordingly, if the potential of blockchain technology is real, it is crucial to acknowledge the limits of existing attempts at adopting a market-driven approach to the governance of decentralized 
blockchain-based applications. In addition to the technical challenges that still have to be addressed by these emergent technologies, one of the main challenges that yet has to be addressed is how to implement a new layer of trust on top of these trustless infrastructures, so as to enable people to collaborate and engage into trusted interactions, while maintaining the benefits of decentralization.

\section{Conclusion}

If technology can be a powerful tool to promote individual freedoms and autonomy, it cannot-by itself-constitute the basis for a free and decentralized society (Benkler, 2016). The decentralized infrastructure of blockchain technology may well increase the opportunities for people to organize and coordinate themselves in a more open and less hierarchical way, but it does not constitute, as such, a sufficient condition to ensure a sustainable decentralized governance structure.

With the Internet, we have learned that protocols are political (DeNardis, 2009): e.g., they can serve to provide us with privacy or they can deprive us from it. Similarly, with blockchain technology, depending on their technical design and payoff structures, blockchains can either emancipate us from the grips of governments and corporations or they can subject us to a set new of selfexecuting code-based rules, elaborated and controlled by a new set of intermediaries.

It is important not to conflate the notion of decentralization at the infrastructural level with decentralization at the governance level (Agre, 2003). While it is, indeed, difficult to deploy a distributed governance system on top of a centralized infrastructure-because the party who controls the infrastructure inherits the power to influence the system-at the same time, a decentralized infrastructure does not necessarily entails a decentralized governance structure. Quite to the contrary, a decentralized infrastructure is often much weaker than its centralized counterpart. Given the lack of a central authority in charge of managing and protecting it, the system can in fact be more easily co-opted by external forces (Bauwens, 2009).

Accordingly, despite its potential for disintermediation and decentralized cooperation, the social and political implications of blockchain technology are difficult to predict. In particular, it remains unclear whether technological decentralization will lead to a more decentralized and democratic society-one where people can freely organize themselves in an open and decentralized manner, freed from the control of governments and corporations-or whether it will instead lead to a more authoritarian society-one where most of our social interactions and economic transactions are mediated by trustless technological systems controlled by a few dominant players.

Once again, an analogy with the Internet may prove useful. Initially perceived as a means to promote individual freedom and autonomy, when powerful economic interests have entered the scene, the Internet eventually evolved into a highly concentrated network, ultimately controlled by governments and corporations (Leamer \& Storper, 2001). If left to its own demise, the evolution of blockchain-based applications might follow the same path.

\section{REFERENCES}

Agre, P. E. (2003). P2P and the promise of internet equality. Communications of the ACM, 46(2), 39-42. Antonopoulos, A. M. (2014). Mastering Bitcoin: unlocking digital cryptocurrencies. " O'Reilly Media, Inc." 
Assange, J., Appelbaum, J., Müller-Maguhn, A., \& Zimmermann, J. (2012). Cypherpunks: Freedom and the Future of the Internet. OR books.

Atzori, Marcella. "Blockchain technology and decentralized governance: Is the state still necessary?." (2015). (December 1, 2015). Available at SSRN

Barrett, N. (1996). The state of the cybernation: Cultural, political and economic implications of the Internet. Kogan Page Limited.

Barlows, John Perry. Declaration of Independence of the Cyberspace. Davos, February 6, 1996

Bauwens, M. (2009). Class and capital in peer production. Capital \& Class, 33(1), 121-141

Benkler, Y. (2016). Degrees of freedom, dimensions of power. Daedalus, 145(1), 18-32

Botsman, R., \& Rogers, R. (2010). What's mine is yours. The rise of collaborative consumption.

Brabham, D. C. (2012). The myth of amateur crowds: A critical discourse analysis of crowdsourcing coverage. Information, Communication \& Society, 15(3), 394-410

Brabham, D. C. (2013). Crowdsourcing. Mit Press

Brito, J., \& Castillo, A. (2013). Bitcoin: A primer for policymakers. Mercatus Center at George Mason University.

Campbell, J. E., \& Carlson, M. (2002). Panopticon. com: Online surveillance and the commodification of privacy. Journal of Broadcasting \& Electronic Media, 46(4), 586-606.

Castells, M. (1996). The network society (Vol. 469). Oxford: Blackwell

De Filippi, P. "Bitcoin: a regulatory nightmare to a libertarian dream." Internet Policy Review 3, no. 2 (2014).

De Filippi, P. (2014). Ubiquitous Computing in the Cloud: User Empowerment vs. User Obsequity. In User Behavior in Ubiquitous Online Environments (pp. 44-63). IGI Global.

De Filippi, P., \& Loveluck, B. (2016). The invisible politics of Bitcoin: governance crisis of a decentralised infrastructure. Internet Policy Review, 5(3).

Deibert, R., \& Rohozinski, R. (2010). Liberation vs. control: The future of cyberspace. Journal of Democracy, 21(4), 43-57.

DeNardis, L. (2009). Protocol politics: The globalization of Internet governance. Mit Press

Diamond, L. (2010). Liberation technology. Journal of Democracy, 21(3), 69-83.

Diamond, L., \& Plattner, M. F. (2012). Liberation technology: Social media and the struggle for democracy. JHU Press.

Drescher, D. (2017). Seeing the Limitations. In Blockchain Basics (pp. 205-211). Apress.

DuPont, Q. (2014). The politics of cryptography: Bitcoin and the ordering machines. Journal of Peer Production, 1(4).

Gale, D. (1955). The law of supply and demand. Mathematica scandinavica, 155-169.

Gervais, A., Karame, G. O., Capkun, V., \& Capkun, S. (2014). Is Bitcoin a Decentralized Currency?. IEEE Security \& Privacy, 3(12), 54-60.

Gervais, A., Karame, G. O., Wüst, K., Glykantzis, V., Ritzdorf, H., \& Capkun, S. (2016, October). On the security and performance of proof of work blockchains. In Proceedings of the 2016 ACM SIGSAC Conference on Computer and Communications Security (pp. 3-16). ACM

Giovannetti, E., \& Kagami, M. (2003). The Internet revolution: a global perspective (Vol. 66). Cambridge University Press.

Goldsmith, J., \& Wu, T. (2006). Who controls the Internet?: illusions of a borderless world. Oxford University Press.

Greenwald, G., MacAskill, E., \& Poitras, L. (2013). Edward Snowden: the whistleblower behind the NSA surveillance revelations. The Guardian, 9(6).

Hawlitschek, Florian; Teubner, Timm; Weinhardt, Christof (2016). "Trust in the Sharing Economy". Swiss Journal of Business Research and Practice. 70 (1): 26-44

Kazeroun, M. (2014). Digital Labour: The Internet as Playground and Factory

Klusácek, J. (2014). Trusted Timestamp in Cryptocurrency Block Chain. Student EEICT

Kroll, J. A., Davey, I. C., \& Felten, E. W. (2013, June). The economics of Bitcoin mining, or Bitcoin in the presence of adversaries. In Proceedings of WEIS (Vol. 2013)

Landau, Susan. "Making sense from Snowden: What's significant in the NSA surveillance revelations." IEEE Security \& Privacy 11, no. 4 (2013): 54-63. 
Lansky, D., Bass, G. D., Ayres, I., Benson, L., \& Radin, B. A. (2007). Data-driven policy. Issues in Science and Technology, 24(1), 11-16

Leamer, E. E., \& Storper, M. (2001). The economic geography of the internet age. Journal of international business studies, 32(4), 641-665

Lévy, P. (2000) World philosophie. Le marché, le cyberespace, la conscience, Paris, Odile Jacob.

Lewenberg, Y., Bachrach, Y., Sompolinsky, Y., Zohar, A., \& Rosenschein, J. S. (2015, May). Bitcoin mining pools: A cooperative game theoretic analysis. In Proceedings of the 2015 International Conference on Autonomous Agents and Multiagent Systems (pp. 919-927). International Foundation for Autonomous Agents and Multiagent Systems

Litman, J. (2001). Digital copyright. Prometheus books

Lyon, D. (1998). The world wide web of surveillance: The internet and off- world power- flows. Information Communication \& Society, 1(1), 91-105.

Lyon, D. (2014). Surveillance, Snowden, and big data: Capacities, consequences, critique. Big Data \& Society, $1(2)$,

Mattila, J. (2016). The Blockchain Phenomenon-The Disruptive Potential of Distributed Consensus Architectures (No. 38). The Research Institute of the Finnish Economy

Milani, Fredrik, Luciano García-Bañuelos, and Marlon Dumas. "Blockchain and business process improvement." BPTrends newsletter (October 2016) (2016

Morozov, E. (2012). The net delusion: The dark side of Internet freedom. PublicAffairs

Nakamoto, S. (2008). Bitcoin: A peer-to-peer electronic cash system.

Oram, A. (2001). Peer-to-Peer: Harnessing the power of disruptive technologies. " O'Reilly Media, Inc."

Posner, R. (1974). Theories of Economic Regulation. Bell Journal of Economics, 5(2), 335-358.

Prisco, G. (2015). Estonian Government Partners with Bitnation to Offer Blockchain Notarization Services to EResidents. Bitcoinmagazine. Com.

Raymond, E. S. (2001). The Cathedral \& the Bazaar: Musings on linux and open source by an accidental revolutionary. " O'Reilly Media, Inc.".

Rosenzweig, R. (1998). Wizards, bureaucrats, warriors, and hackers: Writing the history of the Internet. The American Historical Review, 103(5), 1530-1552.

Sapirshtein, A., Sompolinsky, Y., \& Zohar, A. (2015). Optimal selfish mining strategies in bitcoin. arXiv preprint arXiv:1507.06183.

Schalken, K. (1998). Internet as a New Public Sphere for Democracy (pp. 159-174). Amsterdam: IOS Press

Scott, B. (2015). Visions of a techno-Leviathan: the politics of the Bitcoin blockchain.

Snow, P., Deery, B., Lu, J., Johnston, D., Kirby, P., Sprague, A. Y., \& Byington, D. (2014). Business processes secured by immutable audit trails on the blockchain

Spielman, A. (2016). Blockchain: digitally rebuilding the real estate industry (Doctoral dissertation, Massachusetts Institute of Technology).

Stigler, G. J. (1971). The theory of economic regulation. The Bell journal of economics and management science, 3-21.

Swan, M. (2015). Blockchain: Blueprint for a new economy. " O'Reilly Media, Inc."

Tapscott, D., \& Tapscott, A. (2016). Blockchain Revolution: How the Technology Behind Bitcoin Is Changing Money, Business, and the World. Penguin.

The Economist, “The Trust Machine”, The Economist, Volume 417, Number 8962, October 31st 2015.

Vogel, N. (2015). The Great Decentralization: How Web 3.0 Will Weaken Copyrights. J. Marshall Rev. Intell. Prop. L., 15, 136.

Weber, S. (2004). The success of open source. Harvard University Press.

Wright, Aaron, and Primavera De Filippi. "Decentralized blockchain technology and the rise of lex cryptographia." (2015)

Yermack, D. (2017). Corporate governance and blockchains. Review of Finance, rfw074.

Ziccardi, G. (2012). Resistance, liberation technology and human rights in the digital age (Vol. 7). Springer Science \& Business Media 